\title{
ESTUDO E COMPOSIÇÃO DA AVIFAUNA DO CAMPUS DA FAFRAM- ITUVERAVA, SP
}

SILVA, Rangel Rodrigues de Souza ${ }^{1}$

MORETE, Cintia Natane ${ }^{2}$

Maso, Marislei ${ }^{3}$

RESUMO: O estudo determinou a composição da avifauna do Campus da FAFRAM, bem como a dieta alimentar das espécies e sua frequência de ocorrência (FO\%). A avifauna foi estudada durante 10 meses, maio e agosto de 2011 e posteriormente de dezembro de 2011 a julho de 2012. Foi feito um levantamento onde 36 visitas foram efetuadas, resultando em 73 horas amostrais, duas vezes por semana. As visitas no período matutino eram iniciadas nas primeiras horas do dia (das $6 \mathrm{~h}$ às $8 \mathrm{~h}$ ), no período vespertino era iniciado ao entardecer (das $16 \mathrm{~h} 30 \mathrm{~m}$ às $19 \mathrm{~h}$ ) e no período noturno (das $20 \mathrm{~h}$ às $21 \mathrm{~h}$ ). Com o auxilio de um guia de campo e de um binóculo Tasco Essential $10 X 50$ foi possível registrar 100 espécies de aves, distribuídas em 19 ordens e agrupadas em 38 famílias (22 nãopasseriformes e 16 passeriformes), predominaram as aves insetívoras (34 espécies), seguida das espécies onívoras (32 espécies). Algumas aves registradas possuem hábitos específicos para sua sobrevivência, o que mostra a boa qualidade do ambiente dentro da área de estudo.

Palavras-Chave: Aves. Área de Reserva Legal. Área de Preservação Permanente.

SUMMARY: The study determined the composition of the avifauna of the FAFRAM Campus, as well as the food diet of the species and their incident frequency (IF \%). The avifauna was studied during teen months, from May and August 2011 and subsequently from December 2011 to July 2012. A survey was conducted where 36 visits were carried out whose result was 73-hour samples twice a week. The visits in the morning started in the first hour of the day (from 6 to 8 a.m.), in the afternoon it started at dusk (from 4:30 to 7:00 p.m.) and in the evening (from 8:00 to 9:00 p.m.). With the field guide's help and the use of an binoculars Essential Tasco 10X50 it was possible to register 100 species of birds, distributed among 19 orders and gathered on 38 families (22 no-passeriforms and 16 passeriforms), the insectivorous birds predominated (34 species), followed by the omnivorous species (32 species). Some registered birds have specific habits to survive, which shows the good quality of the environment inside the study area.

Keywords: Birds. Legal Reserved Area. Permanent Preservation Area.

\section{INTRODUÇÃO}

As aves constituem um dos grupos mais bem estudados do ponto de vista ecológico e taxonômico, e são comumente utilizadas como bioindicadores, e na identificação de áreas de endemismo e daquelas prioritárias para conservação (EKEN, 2004 apud OLMOS, 2005).

De acordo com Boçon (2010), de todas as comunidades de vertebrados terrestres as aves são consideradas bioindicadoras, devido a sua ampla distribuição geográfica, pela facilidade de serem encontradas, por possuírem hábitos predominantemente diurnos, por apresentarem diversas funções ecológicas dentro de um ecossistema florestal.

\footnotetext{
${ }^{1}$ Acadêmico da Faculdade de Filosofia Ciências /Fundação Educacional de. E-mail: rangel_rodrigues_silva@hotmail.com 
Com os habitats das aves cada vez mais degradados e somando-se o fato de diversas espécies serem endêmicas de determinadas regiões, como no caso da Mata Atlântica, tornam o grupo um dos mais ameaçados do planeta (BROOKS; BALMFORD, 1996 apud MORETE, 2011).

"Alterações de vegetação podem tornar o ambiente natural impróprio para espécies exigentes e com condições específicas para sobreviver" (DONATELLI; COSTA; FERREIRA, 2004).

Boa parte da Região Neotropical é ocupada pelo Brasil, e o mesmo possui uma grande diversidade de biomas que abrigam um alto número de espécies e grau de endemismos (FUSCALDI; LOURES-RIBEIRO, 2008).

De acordo com registros do Comitê Brasileiro de Registros Ornitológicos (CBRO), estima-se que a avifauna brasileira chegue a 1.832 espécies registradas, muitas dessas espécies estão ameaçadas.

Segundo o Centro de Estudos Ornitológicos (CEO), tem-se registrado para o Estado de São Paulo 808 espécies de aves. Algumas destas não mais ocorrem no estado, outras têm suas populações em tamanhos reduzidos e são ocasionais ou estão ameaçadas de extinção. Por conta do intenso processo de desmatamento e urbanização pelo qual o estado passou, poupando praticamente as regiões montanhosas da Serra do Mar na região litorânea, e da Serra da Mantiqueira, no nordeste do estado, houve uma grande mudança na composição da avifauna, extinguindo algumas espécies, reduzindo a área de ocorrência de outras e permitindo a invasão por dispersão de espécies de espaços abertos (CEO, 2011).

De acordo com Marini; Garcia (2005) a principal ameaça para as aves brasileiras é a perda e a fragmentação de habitats. Para 111 (89,5\%) das 124 espécies brasileiras presentes na lista vermelha da União Internacional para a Conservação da Natureza (IUCN, 2004), a perda e degradação do habitat é uma das principais ameaças, seguida pela captura excessiva (35,5\%). Outras ameaças incluem a invasão de espécies exóticas e a poluição (14\%), a perturbação antrópica e a morte acidental (9,5\%), alterações na dinâmica das espécies nativas $(6,5 \%$ cada), desastres naturais $(5 \%)$ e perseguição $(1,5 \%)$.

O levantamento faunístico é um exercício em que uma série de observações tem por objetivo, apenas catalogar as espécies que existem em certa região (HELLAWELL, 1991 apud PEIXOTO; BATISTA JR; RODOVALHO, 2007).

O levantamento de espécies em ambientes fragmentados está diretamente relacionado com a preservação de certas espécies nesses ambientes. Muitas vezes as espécies de aves mais especializadas, ou seja, aquelas que possuem hábitos alimentares específicos correm mais riscos de desaparecerem em determinados ambientes fragmentados onde predomina as espécies generalistas, ou seja, aquelas que possuem hábito alimentar variável (D’ANGELO NETO et al., 1998).

\section{MATERIAL E METODO}

\section{1 Área de estudo}

A área de estudo está localizada no Campus da Faculdade Dr. Francisco Maeda localizada no município de Ituverava, SP, (20²0’31'S, 4746’07’W e altitude de 619m).

Segundo Carrer e Garcia (2007) o clima para a cidade de Ituverava é considerado do tipo AW Megatérmico (tropical úmido), característico de savanas tropicais com verão úmido e inverno seco e ameno, sendo a temperatura do mês mais frio superior a $18^{\circ} \mathrm{C}$.

A FAFRAM é uma instituição de ensino superior, localizada na área rural do município, mantida da Fundação Educacional de Ituverava.

A área de estudo possui uma área de reserva legal em transição com a área antrópica, seguida de uma Área de Preservação Permanente (APP), que tem como cobertura vegetal primitiva correspondente à floresta Estacional Semidecidual, encontrando também trechos de cerrado, correspondendo à área de 
tensão ecológica na qual se dá o contato do cerrado com a floresta Ombrófila, abrigando importantes remanescentes de campo e mata ciliar (PEREIRA, 2010 apud MORETE, 2011).

\subsection{Levantamento da avifauna}

O método de levantamento foi o quali-quantitativo que tem a finalidade de estabelecer uma listagem da avifauna da área de estudo e sua frequência de ocorrência.

As observações foram feitas com o auxílio de um binóculo Tasco Essential $10 \times 50 \mathrm{~mm}$, e a identificação das aves foi realizada através de literatura específica (TOMAS SIGRIST, 2009; WIKIAVES, 2012). Foi utilizado um caderno de campo para o registro de todas as espécies encontradas. Registros fotográficos foram feitos quando possível com uma câmera digital Sony Cyber-shot DSCW110.

A ordem taxonômica das famílias e a nomenclatura das espécies foram baseadas nas listas do Comitê Brasileiro de Registros Ornitológicos (CBRO, 2011).

\subsection{Levantamento quali-quantitativo}

O levantamento quali-quantitativo foi efetuado nos meses de maio e agosto de 2011 e posteriormente de dezembro de 2011 a julho de 2012. A área de estudo foi amostrada semanalmente, no período da manhã com início às $06 \mathrm{~h} 00 \mathrm{hs}$ sendo finalizadas às $08 \mathrm{~h} 00 \mathrm{hs}$. No período da tarde as observações tiveram início por volta das $16 \mathrm{~h} 30 \mathrm{~min}$ sendo finalizadas por volta das $19 \mathrm{~h} 00 \mathrm{hs}$, e a noite com início por volta das $20 \mathrm{~h} 00 \mathrm{hs}$ sendo finalizadas às $21 \mathrm{~h} 00$.

A área de estudo foi dividida em 11 pontos fixos sendo esses:

Ponto 1: Jardim do Laboratório.

Ponto 2: Borda da Mata perto da entrada antiga .

Ponto 3: Estufas.

Ponto 4: Casa do diretor.

Ponto 5: Campo aberto (pastos).

Ponto 6: Estrada e borda da lagoa.

Ponto 7: Fragmento de Mata (atrás da lagoa).

Ponto 8: Fragmento de Mata (atrás da suinocultura).

Ponto 9: Estrada e jardins (atrás do hospital veterinário).

Ponto 10: Borda da Mata perto das salas de aula.

Ponto 11: Jardins de entrada do Campus.

Através do levantamento quali-quantitativo foi possível calcular a frequência de ocorrência (FO\%) das espécies dentro da área de estudo e sua riqueza (número de espécies encontradas).

$$
\mathrm{FO} \%=\frac{\mathrm{Ndi}}{\mathrm{Nts}} \times 100
$$

$\mathrm{FO} \%$ = frequência de ocorrência, em porcentagem.

$\mathrm{Ndi}=$ número de visitas nas quais a espécie ‘i’ foi observada

$\mathrm{Nts}=$ número total de visitas

Para cada espécie foi designada uma categoria de abundância segundo sua frequência de ocorrência nos censos feitos em um habitat específico segundo NAKA et al. (2002) apud Rodrigues et 
al. (2005): Abundantes (de 75\% e 100\%), Frequente (de 50 a 74\%), Comuns (de 25 a 49\%), Raras (de 24 e $6 \%)$ e Ocasionais $(<5 \%)$.

\subsection{Categorias tróficas}

Sem levar em conta à relação taxonômica, as espécies foram agrupadas em categorias segundo o princípio de guilda, definida como um grupo de espécies que apresentam papel semelhante na comunidade (ODUM, 1988 apud LAPATE, 2006), neste caso o hábito alimentar.

As Categorias tróficas foram as seguintes:

- insetívoros: alimentação baseada em insetos e outros artrópodes.

- onívoros: alimentação baseada em insetos, outros artrópodes e frutos.

- frugívoros: alimentação baseada em frutos.

- granívoros: alimentação baseada em grãos.

- nectarívoros: alimentação baseada em néctar e pequenos artrópodes.

- carnívoros: alimentação baseada em vertebrados vivos.

- piscívoros: alimentação baseada em peixes.

- detritívoros: alimentação baseada em vertebrados mortos.

\section{RESULTADOS}

\subsection{Riqueza específica}

A área de estudo foi visitada semanalmente, de uma a duas vezes por semana, durante oito meses, totalizando 36 visitas, resultando em 73 horas amostrais. Neste período foram registradas 100 espécies de aves, distribuídas em 19 ordens, agrupadas em 38 famílias (22 não-passeriformes e 16 passeriformes). A lista completa das aves encontra-se na Tabela 1.

Tabela 1. Lista das aves registradas no Campus da FAFRAM, Ituverava-SP, Nome científico, Dieta: (SICK, 1984); (MOTTA-JUNIOR,1990); (WIKIAVES, 2012); (SIGRIST, 2009); (LAPATE, 2006). CAR: Carnívoros INS: Insetívoros, PS: Piscívoros, ONI: Onívoros, DET: Detritívoros, NEC: Nectarívoros, GRA: Granívoros .

(Continua)

\begin{tabular}{lc}
\hline Espécie & Dieta \\
\hline & \\
Rupornis magnirostris & CA \\
Dendrocygna viduata & ONI \\
Amazonetta brasiliensis & PS \\
Amazilia versicolor & NEC \\
Eupitomena macroura & NEC \\
Nyctibius griséus & INS \\
Vanellus chilensis & ONI \\
Jacana jacana & ONI \\
Coragyps atratus & DET \\
Columbina squamata & GRA
\end{tabular}


Tabela 1. Lista das aves registradas no Campus da FAFRAM, Ituverava-SP, Nome científico, Dieta: (SICK, 1984); (MOTTA-JUNIOR,1990); (WIKIAVES, 2012); (SIGRIST, 2009); (LAPATE, 2006). CAR: Carnívoros INS: Insetívoros, PS: Piscívoros, ONI: Onívoros, DET: Detritívoros, NEC: Nectarívoros, GRA: Granívoros .

(Continuação)

Columbina talpacoti

GRA

Patagioenas picazuro

GRA

Zenaida auriculata

GRA

Chloroceryle amazona PS

Crotophaga ani

INS

Guira guira

INS

Caracara plancus

CAR

Falco femoralis

CAR

Milvago chimachima

CAR

Aramides cajanea

ONI

Pardirallus nigricans

ONI

Galbula ruficauda

INS

Ardea alba

PS

Ardea cocoi

PS

Bubucus íbis

INS

Butorides striata

PS

Egretta thula

PS

Nycticorax nycticorax

PS

Syrigma sibilatrix

INS

Trigsoma lineatum

PS

Mesembrinibis cayennensis

ONI

Theristicus caudatus

ONI

Ramphastos toco

ONI

Campephilus melanoleucos

INS

Colaptes campestris

INS

Colaptes melanochloros

INS

Dryocopus lineatus

INS

Picumus cirratus

INS

Velinornis passerinus

INS

Aratinga aurea

FRU

Aratinga auricapilus

FRU

Aratinga leucophthalma

FRU

Brotogeris chiriri

FRU 
Tabela 1. Lista das aves registradas no Campus da FAFRAM, Ituverava-SP, Nome científico, Dieta: (SICK, 1984); (MOTTA-JUNIOR,1990); (WIKIAVES, 2012); (SIGRIST, 2009); (LAPATE, 2006). CAR: Carnívoros INS: Insetívoros, PS: Piscívoros, ONI: Onívoros, DET: Detritívoros, NEC: Nectarívoros, GRA: Granívoros .

(Continuação)

Forpus xanthopterygius

FRU

Orthopsicattaca mamilata

FRU

Lepdocolaptus angustirostris

INS

Donacrobius atricapilla

INS

Sicalis flaveola

GRA

Sicalis luttola

GRA

Sporophila caerulescins

GRA

Volatina jacarina

GRA

Zonotrichia capensis

GRA

Furnarius rufus

INS

Phacelodomus ruber

INS

Progne tapera

INS

Pygochelidon cyanoleuca

INS

Chrysomus ruficapillus

ONI

Icterus pyrrhopterus

ONI

Molothrus bonariensis

ONI

Passer domesticus

ONI

Polioptila dumicola

INS

Todirostrum cinereum

INS

Taraba major INS

Thamnophilus doliatus INS

Nemosia pileata

ONI

Tangara cayana

ONI

Tangara palmarium

ONI

Tangara sayaca

ONI

Tersina viridis

ONI

Saltator similis

ONI

Troglodytes musculus

INS

Mimus saturninus

ONI

Turdus amaurochalinus

ONI

Turdus leucomelas

ONI

Arundinicola leucocephala

INS 
Tabela 1. Lista das aves registradas no Campus da FAFRAM, Ituverava-SP, Nome científico, Dieta: (SICK, 1984); (MOTTA-JUNIOR,1990); (WIKIAVES, 2012); (SIGRIST, 2009); (LAPATE, 2006). CAR: Carnívoros INS: Insetívoros, PS: Piscívoros, ONI: Onívoros, DET: Detritívoros, NEC: Nectarívoros, GRA: Granívoros .

(Conclusão)

Arremon flavirostris

Empidomonus varius

Elaenia flavogaster

Fluvicola nengeta

Machetornis rixosa

Megarynchus pitangua

Myiarchus ferox

Myiodynastes maculatus

Myozetetes similis

Pitangus sulphuratus

Tyrannus melancholicus

Tyrannus savana

Cyclarhis gujanensis

Phalacrocorax brasilianus

Athene cunicularia

Megascopis choliba

Tyto alba

Crypturellus ssp

Amazilia fimbriata

Amazilia láctea

Philohydor lector

Icterus cayanensis

Dacnis cayana

Euphonia chlorotica

\section{INS}

INS

ONI

INS

INS

ONI

INS

ONI

ONI

ONI

INS

INS

ONI

PS

INS

INS

CAR

ONI

NEC

NEC

ONI

ONI

FRU

ONI

Fonte: Elaborado pelos autores.

A ordem que se apresentou com maior número de famílias foi a ordem dos Passeriformes (15 famílias) seguida diretamente das ordens dos não-passeriformes, Pelecaniformes, Piciformes, e Charadriformes (duas famílias cada).

A família da ordem dos passeriformes que apresentou um maior número de indivíduos foi a familia Tyrannidae (13 espécies), seguida da família Emberizidae e Thraupidae (6 espécies cada).

A família das ordens dos não-passeriformes que apresentou um maior número de espécies foi a família Ardeidae (8 espécies), seguida da família Piscitacidae e Picidae (6 espécies cada) e Columbidae (4 espécies). 
As espécies de aves foram agrupadas de acordo com a sua categoria alimentar. A categoria que se apresentou em maior número foi a insetívora com 32 espécies, seguida da onívora com 27 espécies, Piscívora e Granívora com 10 espécies respectivamente. (Gráfico 1.)

Gráfico 1. Distribuição das espécies de aves registradas no Campus da FAFRAM (Ituverava -SP) de acordo com sua categoria trófica (Calculado de acordo com o $n^{\circ}$ de espécies)

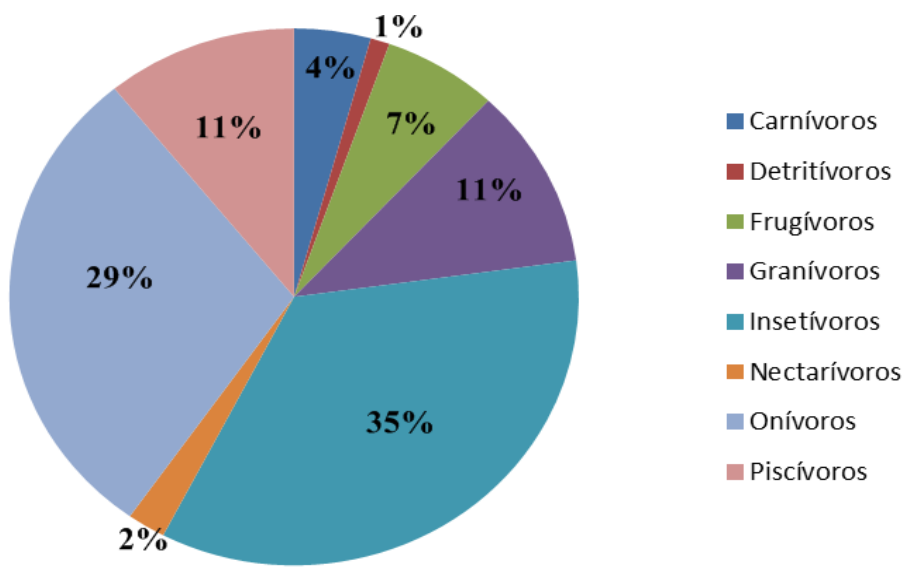

Fonte: Elaborado pelos autores

\subsection{Frequência de ocorrência}

As espécies de aves foram classificadas de acordo com sua frequência dentro da área de estudo durante todo o período amostral. Das 100 espécies encontradas dentro da área, nenhuma foi considerada como abundante ou frequente, 55 foram consideradas raras, 19 foram consideradas comuns e 23 foram consideradas ocasionais sendo que 16 espécies desse total foram registradas apenas uma vez durante todas as visitas.

Pitangus sulphuratus e Tyrannus melancholicus ambas pertencentes à ordem dos passeriformes (Familia Tyrannidae), Furnarius rufus e Passer domesticus também pertencentes à ordem dos passeriformes (Famílias: Furnaridae e Passeridae respectivamente) foram considerados dentro da categoria de FO\% (Frequência total por ocorrência) como comuns, tendo uma maior porcentagem dentro desta categoria.

Aratinga aurea, Brotogeris chiriri, Aratinga leucophthalma pertencentes às ordens dos nãopasseriformes (Ordem: Psitaciformes, família Psitacidae), foram considerados comuns dentro da categoria de $\mathrm{FO} \%$, juntamente com algumas outras espécies que tiveram uma porcentagem menor, porém se enquadram dentro dessa mesma distribuição.

Falco femoralis, Rupornis magnirostris pertencentes às ordens dos não-passeriformes (Ordens: Falconiformes e Accipitriformes, famílias: Falconidae e Accipitridae respectivamente) e Arremon flavirostris, Sicalis luteola pertencentes a ordem dos passeriformes (Famílias: Tyrannidae e Emberizidae respectivamente) foram considerados ocasionais dentro da categoria de FO\% tendo sido registrados apenas de 2 a 3 vezes durante toda pesquisa.

Algumas espécies como Dendrocygna viduata, Amazonetta brasiliensis, Forpus xanthopterygius, Orthopsicattaca mamilata (Não-passeriformes, famílias: Anatidae e Psitacidae) e algumas outras espécies ocasionais foram registradas apenas uma vez durante todo o período de amostra. 


\section{DISCUSSÃ̃}

Segundo Carvazere; Moraes; Donatelli (2009) "nos últimos 30 anos alguns trabalhos sobre a avifauna das matas semidecíduais foram realizados no interior do estado de São Paulo, e o conhecimento de algumas localidades é bastante compreendido."

De acordo com Barros; Paranhos; Roma (1989) para obter um número considerável de espécies registradas é necessário uma estabilidade de no mínimo 200 horas amostrais e mesmo ao atingir essa quantidade de horas pode ser registrado novas espécies que até então não tinha sido encontradas.

Portanto, o presente trabalho poderia não estabelecer a curva de acúmulo de espécies mesmo com um esforço amostral de 200 horas, porém o estudo permitiu analisar com apenas 73 horas amostrais que o Campus da FAFRAM, Ituverava,SP, possui uma aprazível composição avifaunistica.

Segundo Donatelli; Costa; Ferreira (2004), “o ambiente natural pode se tornar impróprio para determinadas espécies de aves que exigem condições específicas para sua sobrevivência”, essas aves são consideradas especialistas. Foram encontradas espécies com essas características, como o Anatídeo Trigsoma lineatum (Socó-boi), que possui uma forte ligação com corpos d'água, por causa do seu tipo de dieta que é baseada em peixes e pequenos crustáceos. (Sick, 1989)

Assim como existem espécies que não se adaptam as alterações ambientais, existem espécies que se beneficiam e respondem a essas alterações, como é o caso do tyrannideo Pitangus sulphuratus (Bem-te-vi) (COLLAR et. al, 1997 apud MARINI; GARCIA, 2005). Concordando com o autor $P$. sulphuratus foi registrado em todos os ambientes desde o campo aberto e matas fechadas ao fragmento de mata ciliar ligada ao açude, essa espécie de ave teve um grande registro de ocorrência durante toda a pesquisa sendo encontrado em todos os ambientes, o que mostra sua facilidade de adaptação.

A categoria alimentar com mais espécies foi a insetívora, seguida por onívora, granívora, frugívora e piscívora (ambas com a mesma quantidade de espécies), carnívora, nectarívora e por último detritívoras. Discordando assim de Donatelli et al.(2004) que também obtiveram maior porcentagem de aves insetívoras, porém, seguidas de frugívoras, onívoras, carnívoras, nectívoras e detritívoras, o que seria o ideal para áreas de matas tropicais preservadas.

O estudo da composição regional da biota é essencial para o conhecimento das espécies que se encontram em determinados tipos de ambiente, esse tipo de estudo é de extrema importância para o conhecimento biológico das espécies e a criação de novas áreas de preservação e conservação de algumas espécies, que acabam não tendo uma boa representação. (FERREIRA; COSTA; RODRIGUES, 2009).

Isso torna o Campus da FAFRAM muito importante como qualquer outra área de preservação dentro da comunidade biológica por possuir uma área bem preservada composta de uma vasta biodiversidade.

\section{CONCLUSÃO}

De acordo com o presente estudo a avifauna do Campus da FAFRAM possui uma riqueza considerável, onde as famílias tyrannidae (Passeriformes) e Ardeidae (Não-passeriformes) foram as mais abundantes dentro da área de estudo. Nenhuma espécie encontrada está considerada ameaçada de extinção. 


\section{REFERÊNCIAS}

BARROS, Y. de M.; PARANHOS, S. J.; ROMA, J. C.. Levantamento preliminar das aves da Fazenda Santa Carlota - Municipio De Cajuru - (SP), com informações ecológicas sobre as espécies não-passeriformes. 1989. 101 f. Monografia (Bacharel em Ciências Biológicas) - USP, Ribeirão Preto.

BROOKS, T; BALMFORD, A. Atlantic forest extinctions. In: MORETE, Cintia Natane. A avifauna do Campus II da Fundação Educacional De Ituverava, SP.2011. Trabalho De Conclusão De Curso (Licenciatura em Ciências Biológicas), Faculdade de Filosofia Ciências e Letras de Ituverava.

BOÇON, R.. Riqueza e abundância de aves em três estágios sucessionais da floresta ombrófila densa submontana, antonina, Paraná. 2010. 104 f. Tese (Doutorado) - Universidade Federal Do Paraná, Curitiba. Disponível em:

$<$ http://dspace.c3sl.ufpr.br/dspace/bitstream/handle/1884/26214/tese\%20roberto\%20bocon.pdf?sequence $=1>$. Acesso em: 15 abr. 2012.

CARRER, T. T.; GARCIA, a . Classificação climática para a cidade de Ituverava/SP. Nucleus, Ituverava, v. 4, n. 1-2, p.29-38, set. 2007. Disponível em:

http://www.nucleus.feituverava.com.br/index.php/nucleus/article/view/9/36. Acesso em: 15 abr. 2012.

CAVARZERE, V.; MORAES, G. Parmezani; DONATELLI, R. J.. Avifauna da estação Ecológica dos Caetetus, Interior de São Paulo, Brasil. Papéis Avulsos de Zoologia. São Paulo. v.49, n.35, p.477-485, dez. 2009.

CENTRO DE ESTUdOS ORNITOLÓGICOS. Avifauna do Estado de São Paulo. Disponível em $<$ http://www.ceo.org.br >. Acesso em 25 jan.2012.

COMITÊ BRASILEIRO DE REGISTROS ORNITOLÓGICOS. Listas das aves do Brasil. 10. ed. Disponível em: <http://www.cbro.org.br>. Acesso em: 25 jan.2012.

COLLAR, N. J. D. Predicts the Number of Threatened Birds in Insular Southeast Asia. In: MARINI, Miguel Â.; GARCIA, F. I.. Conservação de aves no Brasil. Megadiversidade, Brasilia, v. 1, n. 1, p.95102, jul. 2005. Disponível em:

<http://www.conservation.org.br/publicacoes/files/14_Marini_Garcia.pdf>. Acesso em: 12 ago. 2012.

D'ANGELO NETO, S.et al. Avifauna de quatro fisionomias florestais de pequeno tamanho (5-8 ha) no Campus da UFLA. Revista Brasileira de Biologia, Rio de Janeiro, v.58, n.3, p. 463-472, 28 ago. 1998.

DONATELLI, R. J.; COSTA, T. V. V.; FERREIRA, C. D.. Dinâmica da avifauna em fragmento de mata da Fazenda Rio Claro, Lençóis Paulista, São Paulo, Brasil. Revista Brasileira de Zoologia. Lavras, v.21, n.1, p.97-114, mar. 2004

EKEN, G. et. al. Key Biodiversity Areas as Site Conservation Targets. In: OLMOS, Fábio. Aves ameaçadas, prioridades e políticas de conservação no Brasil. Ponto de Vista: Natureza \& Conservação, Brasil, v. 3, n. 1, p.21-42, abr. 2005.

FERREIRA, Jordana Demicheli; COSTA, Lilian Mariana; RODRIGUES, Marcos. Aves de um remanescente florestal do Quadrilátero Ferrifero, Minas gerais. Biota Neotropica. v.9, n.3, p.39-54, jul. 2009.

FUSCALDI, R. G.; LOURES-RIBEIRO, A.. A avifauna de uma área urbana do município de Ipatinga, Minas Gerais, Brasil. Biotemas, Santa Catarina, v. 3, n. 21, p.125-133, set. 2008. 
GOOGLE EARTH. Foto de Satélite, Localização da cidade de Ituverava no mapa do estado de São Paulo-Brasil, 2004, Foto de satélite. Altitude do ponto de visão 1736.59 m. Acesso em 21 de Maio de 2012.

GOOGLE EARTH. Localização do Campus da FAFRAM no município de Ituverava-SP, 2004, Foto de satélite. Altitude do ponto de visão 2.58 km. Acesso em 21 de Maio de 20012.

HELLAWELL, J.M. Development of a rationale for monitoring. In: PEIXOTO, J. E.; BATISTA JUNIOR, I. S.; RODOVALHO, M. V. de T.. Levantamento da avifauna de áreas da usina vertente utilizado como objeto de avaliação ambiental. Disponível em: $<\mathrm{http} / / /$ sare.unianhanguera.edu.br/index.php/rensc/article/viewFile/320/321>. Acesso em: 12 fev. 2012.

LAPATE, Ma. E.. Levantamento e caracterização da avifauna de uma área reflorestada no Campus da USP de Ribeirão Preto, SP. 2006. 69 f. Monografia (Bacharel em Ciências Biológicas) - USP, Ribeirão Preto.

MARINI, M. Â.; GARCIA, F. I.. Conservação de aves no Brasil. Megadiversidade, Brasilia, v. 1, n. 1, p.95-102, jul. 2005. Disponível em:

<http://www.conservation.org.br/publicacoes/files/14_Marini_Garcia.pdf>. Acesso em: 12 ago. 2012.

MOTTA JÚNIOR, J. C.. Estrutura Trófica e Composição das Avifaunas de Três Habitats Terrestres na Região Central do Estado de São Paulo. Ararajuba, Rio de Janeiro, v. 1, n. , p.65-71, ago. 1990. Disponível em: <http://www.ararajuba.org.br/sbo/ararajuba/artigos/Volume1/ara1art6.pdf >. Acesso em: 15 jul. 2012.

ODUM, W.E. Comparative Ecology of Tidal Freshwater and Salt Marches. In: LAPATE, M. E.. Levantamento e caracterização da avifauna de uma área reflorestada no Campus da USP de Ribeirão Preto, SP. 2006. 69 f. Monografia (Bacharel em Ciências Biológicas) - USP, Ribeirão Preto.

PEREIRA, M. Levantamento de espécies arbóreas de um fragmento de Área de Reserva Legal no Campus da Faculdade Dr. Francisco Maeda - município de Ituverava - SP. In: MORETE, C. N.. A avifauna do Campus II da Fundação Educacional De Ituverava, SP.2011. 1 v. Trabalho De Conclusão De Curso (Licenciatura) - Curso de Ciências Biológicas, Faculdade de Filosofia Ciências e Letras de Ituverava, Ituverava, 2011.

NAKA, L.N et al. Bird conservation on the island of Santa Catarina. In: RODRIGUES, M. et al. Aves do parque Nacional da Serra do Cipó.Revista Brasileira De Zoologia, Belo Horizonte, Minas Gerais, v. 2, n. 22, p.326-338, jun. 2005.

SICK, H. 1984. Ornitologia brasileira. 3.ed. Brasília, UNB, vol.1 431p.

SIGRIST, T.. To the Birds of Brazil: Avifauna Brasileira. Vinhedo - SP: Avis Brasilis, 2009. vol. 2. (Guia de Campo).

WIKIAVES. A Enciclopédia das Aves do Brasil. Disponível em: 〈http://www.wikiaves.com.br/>. Acesso em: 27 abr. 2012. 
